\title{
Supervised Fuzzy C-Means Techniques to Solve the Capacitated Vehicle Routing Problem
}

\author{
Mohamed Shalaby ${ }^{1,2}$, Ayman Mohammed $^{1}$, and Sally Kassem ${ }^{1,2}$ \\ ${ }^{1}$ Smart Engineering Systems Research Center, Nile University, Egypt \\ ${ }^{2}$ Faculty of computers and Artificial Intelligence, Cairo University, Egypt
}

\begin{abstract}
Fuzzy C-Means (FCM) clustering technique is among the most effective partitional clustering algorithms available in the literature. The Capacitated Vehicle Routing Problem (CVRP) is an important industrial logistics and managerial NP-hard problem. Cluster-First Route-Second Method (CFRS) is one of the efficient techniques used to solve CVRP. In CFRS technique, customers are first divided into clusters in the first phase, then each cluster is solved independently as a Traveling Salesman Problem (TSP) in the second phase. This research is concerned with the clustering phase of CFRS, and TSP is then solved using a traditional optimization method. Three supervised FCM based techniques are proposed to solve the clustering phase at reduced cost via centroids (pre-FCM) initialization phase. The proposed pre-FCM initialization techniques are developed to be problem dependent. Hence, three initialization techniques are first developed using K-means technique, spatially equally distributed, and demand weighted center of mass. Then, a modified demand weighted fuzzy c-means objective function is employed to assign customers to clusters. To compare the performance of the proposed supervised FCM techniques, forty-two CVRP benchmark problems are solved using the traditional fuzzy $C$-means algorithm and the developed algorithms. Extensive comparisons are conducted between the traditional fuzzy C-means algorithm, the three proposed initialization techniques, and other fuzzy $C$ means techniques available in the literature. Results show that the proposed three initialization techniques are efficient in terms of solution quality and computational cost.
\end{abstract}

Keywords: Capacitated vehicle routing problem, supervised fuzzy c-means, fuzzy clustering.

Received February 20, 2021; accepted March 7, 2021

https://doi.org/10.34028/iajit/18/3A/9

\section{Introduction}

Partitional clustering methods are widely used because of their simplicity in solving complex combinatorial problems. Partitional clustering methods mostly begin with initial random data assignments and then iteratively reallocate them. This moves data points between clusters until the objective function is optimized [48]. Although partitional clustering methods are of high efficiency, they are not suitable for a huge amount of data [63]. One of the partitional clustering methods is the Fuzzy CMeans (FCM) clustering technique. FCM was initially defined by Dunn [17] and generalized later by Bezdek [6]. Since then, it has been applied in a wide range of both substantive areas and research studies. It permits data points to belong to more than one cluster with varying degrees of membership [68].

The fuzziness parameter $(\mathrm{m})$ or the partition matrix exponent in FCM was proposed by Dunn $[17,18]$ and Bezdek [6]. Although the value for $(\mathrm{m})$ can be any number greater than one, the most chosen value for $(\mathrm{m})$ is $m=2$ [46]. A method to find the optimum value for (m) was proposed by McBratney and Moore [44] for the application of climatic classification. In the same context, Cui et al. [15] argued that the best values for (m) are when (m) is in the range between 1.5 and 2.5. Another study by Choe and Jordan [10] stated that the choice of $(\mathrm{m})$ is strongly related to the complexity of the data under investigation. Different values for (m) were tested ranging between 1 and 40 using fuzzy decision theory introduced by Bellman and Zadeh [5].

The Vehicle Routing Problem (VRP) is an important industrial real-life transportation problem. Most industries and delivery services face this problem when it comes to distributing their products [31]. Due to its complexity and high importance for industrial logistics, the problem has been subject to intensive research since it was defined by Dantzig and Ramser [16].The main objective of solving the classical VRP is to minimize the transportation cost. This is achieved by determining the optimal set of routes for the fleet of vehicles delivering products to customers under some constraints. According to the classical VRP definition, all the vehicles are initially located at a central depot. A vehicle starts its route from the central depot, visits all its predetermined customers, and ends the trip back at the central depot. The total cost is represented by the total accumulated distances traveled by the whole fleet to satisfy all the demand points.

The VRP is a generalized form of the Traveling Salesman Problem (TSP). Each vehicle and its assigned customers can be separated and solved as a TSP, Figure 1 shows the similarity between both problems. 

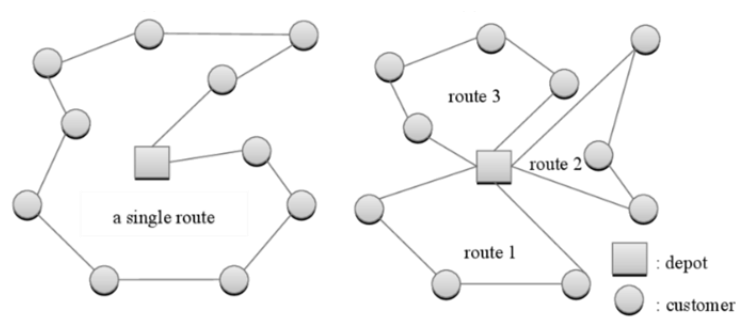

Figure 1. Typical solution of a TSP and VRP.

Several VRP variants are introduced by adding some constraints representing real-life circumstances. For instance, in some cases, the vehicles start their trips from different depots resulting in a multi-depot VRP [45]. Furthermore, some manufacturers collect their used products for proper disposal. This resulted in studying VRP within the context of reverse logistics $[29,33,36$, 62]. Another extension is VRP with a heterogeneous fleet in which vehicles of different sizes are employed for product deliveries [23, 25]. Moreover, periodic VRP is when the demand is required to be fulfilled periodically [64]. Again, split delivery VRP allows fulfilling the demand on multiple deliveries that the demand may exceed the vehicle capacity [2]. In many cases, the problem parameters are stochastic, and hence stochastic VRP has been extensively studied [52, 57, 69]. Moreover, when customers are required to be served within specific time windows results in VRP with time Windows (VRPTW) [55]. Another recognized variant is the Capacitated Vehicle Routing Problem (CVRP). It considers capacity constraints on vehicles that the total demand for a set of customers served by a vehicle does not exceed the vehicle capacity [43, 57]. The mathematical model representing the CVRP [39] is formulated as follows:

$$
\text { Minimize } \mathrm{Z}=\sum_{i=1}^{N} \sum_{j=1}^{N} \sum_{v=1}^{K} C_{i j} x_{i j v}
$$

Subject to:

$$
\begin{gathered}
\sum_{k=1}^{K} \sum_{j=1}^{N} x_{i j k} \leq K, \text { for } \mathrm{i}=0 \\
\sum_{j=1}^{N} x_{i j k}=1, \quad \text { for } i=0 \text { and } k \in\{1, \ldots, K\}
\end{gathered}
$$

$$
\begin{aligned}
& \sum_{\mathrm{j}=1}^{\mathrm{N}} \mathrm{x}_{\mathrm{jik}}=1, \quad \text { for } i=0 \text { and } k \in\{1, \ldots, K\} \\
& \sum_{k=1}^{K} \sum_{j=0, j \neq i}^{N} x_{i j k}=1, \quad \text { for } i \in\{1, \ldots, N\}
\end{aligned}
$$

$$
\sum_{k=1}^{K} \sum_{j=0, i \neq j}^{N} x_{i j k}=1, \quad \text { for } j \in\{1, \ldots, N\}
$$

$$
\sum_{i=1}^{N} d_{i} \sum_{j=0, j \neq i}^{N} x_{i j k} \leq Q, \quad \text { for } i \in\{1, \ldots, N\}
$$

$\mathrm{x}_{\mathrm{ijk}}=\left\{\begin{array}{l}1, \text { if vehicle } k \text { travels from node } i \text { to node } j, \\ 0, \text { otherwise }\end{array}\right.$

$$
I \neq j ; I, j=0,1, \ldots, N ; k=1, \ldots, K .
$$

Where $\mathrm{Z}$ is the objective function minimizing the total routing distance or cost, $N$ is the total number of customers or demand points, $K$ is the total number of vehicles, $Q$ represents the capacity of a vehicle, $C_{i j}$ is the distance between the point $(i)$ and point $(j), d_{i}$ is the demand of the $i^{\text {th }}$ customer. $X_{i j k}$ is a binary decision variable it equals 1 if a vehicle $(k)$ travels from a node (i) to another node $(j)$.

Constraint set (2) is to make sure that the number of routes is equal to the number of vehicles by setting the maximum number of trips leaving the $\operatorname{depot}(i=0)$ equal to the total number of trucks $(K)$. The constraint sets (3) and (4) are to make sure that for each truck there is only one complete route leaving and entering the depot. Furthermore, constraint set (5) is to assure that for each node $i$, only one arc emanates for each truck, while set (6) is to assure that for each node $j$, there is exactly one arc for each vehicle entering to it. This is to make sure that each node is visited only once. Constraint set (7) assures that the total demand assigned to each vehicle does not exceed its capacity.

The vehicle routing problem areas extends to include routes optimization within industrial facilities such as Inventory Routing Problem (IRP). It is considered as a VRP and inventory management problem as it aims to obtain the optimal routes and inventory scheduling simultaneously [60].

Technological advancement allowed collaborative vehicle routing. Online cooperation between the fleet of vehicles leads to a dramatic increase inefficiency and consequently reducing cost [23]. The cooperation may consider capacities, traffic jams, and served customers. For a deeper understanding of the problem view the research by Cruijssen et al. [14] and Guajardo and Rönnqvist [27].

The nature of the vehicle routing problem is proven to be NP-hard [40]. Relatively small instances can be solved using exact methods [13, 46]. However, the computational time is highly fluctuating and requires much effort. Therefore, heuristics and metaheuristics are employed to solve different size instances of the problem and its variants [53].

Cluster-first route-second is one of the methods utilized to solve CVRP, where the clustering phase represents the main step in the heuristic [9]. In the cluster-first route-second method, all demand points are grouped into several clusters. The number of clusters and the number of vehicles should be the same and each cluster must maintain the vehicle capacity constraint. Thus, the clustering phase can be considered as a Capacitated Clustering Problem (CCP). The routing phase takes place right after the clustering phase (route second). Determining routes requires setting the sequence to visit customers, by each vehicle, within a cluster [38].Various clustering approaches have been employed to solve complex VRP to save processing time $[37,56,61]$. Clustering techniques such as partitional, 
density-based, hierarchical, and probabilistic clustering are among the approaches used to solve VRP.

The authors spotted some previous studies that used the fuzzy theory to solve the vehicle routing problem since 1994. For instance, Kagaya et al. [32] considered fuzzy location vehicle routing problem by dividing the trips into groups of two relying on their fuzzy relation properties. Afterward, fuzzy integrals were used for similarity computations between the groups. The determined membership values approve the trips with higher membership. This methodology is considered of high flexibility and efficiency when it comes to high numbers of customers. This technique was used as a first step for solving the VRP using an optimization algorithm.

Jiuh-Biing [31] employed a fuzzy clustering optimization for demand locations in the VRP. The technique considered the problem as of dynamic nature. The proposed technique had multi phases. Firstly, the demand locations should be classified and clustered based on predetermined priorities. After that multiobjective optimization algorithms should be employed to determine the routes. This technique was applied to case studies and improved the performance by $20 \%$.

Yong et al. [70] proposed an optimization algorithm based on three phases. The first phase was transforming the customers' characteristics into a linguistic form. Then the fuzzy integral technique was employed to plot subcriteria using a fuzzy trapezoidal objective function. The second phase is customers' clustering using Fuzzy Sets. Then comes the third phase, dynamic programming for routes' optimization. In the same context, Yalcin and Erginel [67] developed a multi-objective fuzzy algorithm for solving VRP with line hauls and backhauls. The technique consists of three phases, the first phase is concerned with customers' clustering. In this stage, all the vehicles are assigned to demand points using two objective functions. The goal is to minimize the distance to be traveled by the fleet and yet maximize the profit accordingly. The second phase is routes' designs in which the problem is divided into multiple sub problems. The last phase is using a local search algorithm to improve the routes.

Ewbank et al. [20] proposed a fuzzy clustering methodology to accurately analyze and predict fuzziness parameters for the vehicle routing problem. An unsupervised fuzzy learning algorithm based on Fuzzy c-means was proposed to solve the CVRP in a reasonable processing cost. Moreover, clusters' membership values were used to assign the demand points to clusters by considering the vehicle capacity.

Bi et al. [7] integrated fuzzy clustering with a genetic algorithm to improve the initialization phase as a step towards developing an evolutionary algorithm for solving VRP with multiple depots and multi-objectives.

Marinelli et al. [43] used the fuzzy c-means clustering technique to develop a dynamic approach for a twoechelon CVRP. The exact algorithm was used to obtain the optimal routes by dividing the problem into sub problems.

$\mathrm{Xu}$ et al. [66] developed a multi-stage algorithm for the Dynamic Vehicle Routing Problem (DVRP). In the first stage, the dynamic model was designed based on customers' satisfaction levels and cost of operation. In the second stage, the fuzzy C-means clustering technique along with a justifiable granularity principle was integrated to solve the problem. In a more recent study, Shalaby et al. [58] used unsupervised modified FCM algorithm in the clustering phase to solve the classical CVRP. However, the authors did not expose the concept of supervised modified FCM and did not compare the results with original FCM results. Therefore, in this paper, three supervised FCM techniques are proposed by employing an initialization phase. The proposed preFCM initialization techniques are developed to be problem dependent. The three proposed initialization techniques are developed based on K-means technique, spatially equally distributed, and demand weighted center of mass.

This paper is organized as follows: section 2 describes in details the unsupervised FCM techniques and the proposed supervised FCM techniques. The computational experimentation setup is then presented in section 3. In section 4, a comprehensive comparative study is conducted. Forty-two instances are solved to evaluate the efficiency of the proposed techniques. This is by comparing the obtained values and their optimal or best-known values. The advantages, and limitations of such algorithms are discussed. Finally, section 5 concludes the work presented in this paper.

\section{Proposed Methodologies}

The objective of the heuristics presented in this section is to assign the demand nodes or customers to several clusters. Then the optimum route passing all customers within a cluster is determined. This is with the condition that the total demand of each cluster does not exceed the vehicle capacity. It is also assumed that the fleet of vehicles is homogeneous. First, the clustering phase algorithm is illustrated. Afterward, the Traveling Salesman Problem (TSP) model used for the routing phase is presented.

\subsection{Clustering Phase (FCM and Modified FCM)}

Initially, the traditional unsupervised FCM model [6] is used in the clustering phase with adding the clusters' capacity constraints as follows:

$$
\begin{gathered}
\text { Minimize } Z=\sum_{i=1}^{N} \sum_{j=1}^{Y} \mu_{i j}^{m}\left\|x_{i}-C_{j}\right\|^{2} \\
C_{j}=\frac{\sum_{i=1}^{D} \mu_{i j}^{m} x_{i}}{\sum_{i=1}^{D} \mu_{i j}^{m}}
\end{gathered}
$$




$$
\begin{aligned}
& \mu_{i j}=\frac{1}{\sum_{k=1}^{N}\left(\left\|x_{i}-c_{j}\right\|\right)^{\frac{2}{m-1}}} \\
& \sum_{i=1}^{V} D_{i j} \leq Q \quad \forall J \in\{1,2, \ldots . Y\}
\end{aligned}
$$

Where $Z$ represents the objective function of minimizing the distance between the data points and the generated centroids, $N$ is representing the number of customers or demand points, $Y$ is the number of clusters or vehicles, $m$ is the fuzziness parameter for controlling the degree of fuzzy overlap, with $\mathrm{m}>1, x_{i}$ is the $i^{\text {th }}$ data point, $c_{j}$ is the center of the $j^{\text {th }}$ cluster, $\mu_{i j}$ is the degree of membership of $x_{i}$ in the $j^{\text {th }}$ cluster. $D_{i j}$ is the demand of the $i^{\text {th }}$ point in the $j^{\text {th }}$ cluster, $v$ is the number of points within a cluster, $Q$ is the capacity of a single-vehicle.

Unsupervised FCM randomly generates the cluster membership values and assigns the clusters' centers. Then it updates the clusters iteratively each time the objective function is calculated, let's call that the inner loop. The stopping criterion is when the maximum number of inner loop iterations is achieved, or the improvement is less than a pre-specified value. After that, the demand of all data points within a cluster is calculated. If the demand exceeds the capacity of the vehicle, the algorithm starts again until the capacity constraint is satisfied. In other words, there is another type of iterations in the case of violating the vehicle capacity, and let's call that the outer loop.

When traditional unsupervised fuzzy C-means is used for clustering the CVRP instances, it took a relatively high number of outer loop iterations and mostly gave inconvenient results. The traditional unsupervised FCM objective function shown in Equation (8) aims to minimize the distance between data points and the generated centroids regardless of the demand. Adding clusters' capacity constraint in the clustering phase causes high computational cost and inefficient results. Thus, a demand weighted objective function is employed instead of the original objective function as in Equation (12). Making such modifications is completely valid in relevance to previous studies. In this context, many researchers modified the FCM algorithm for different applications such as studies present in $[19,24,30,35]$. The routing phase treats the clusters as separate TSP.

$$
J_{m}=\sum_{i=1}^{D} \sum_{j=1}^{D} \mu_{i j}^{m}\left\|\frac{x_{i}-C_{j}}{D i}\right\|^{2}
$$

The flowchart in Figure 2 represents the followed methodology of using the modified FCM algorithm for solving the CVRP [58]. In the beginning, the number of clusters is introduced as a determined input. It is set to be the number of vehicles dedicated to products delivery. The second input is the co-ordinates of the customers or the demand points. Then the modified FCM is executed to get the clusters and their assigned data points. Then the total demand within each cluster is compared with the vehicle's capacity. This is to make sure all the demand points within a cluster are fulfilled by one vehicle. If the capacity constraint is violated the algorithm will be initialized again with new random centroids until the capacity constraint is satisfied. After that, the depot location is added to each cluster and the problem is solved as multiple TSP. The algorithm was executed using different random values of fuzziness exponent $(\mathrm{m})$ each iteration for each instance, $2 \leq \mathrm{m} \leq$ 35. Each iteration ran 15 times and the lowest output was recorded.

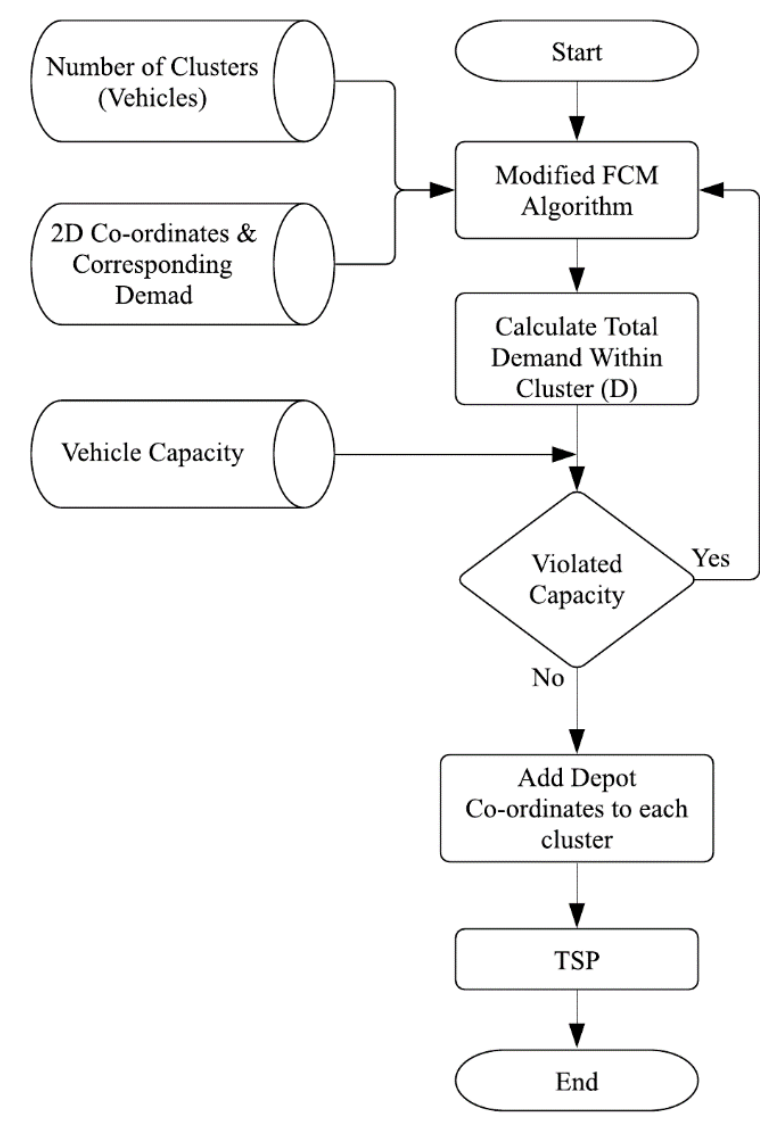

Figure 2. Flowchart of modified FCM methodology [58].

\subsection{Proposed Supervised Fuzzy C-Means}

FCM might require a high computational cost to reach the desired results for several reasons such as the random initialization, the fuzziness parameter value, the number of data points, and the number of inner loop iterations. It is not an easy task to control all these factors especially that some of them are noncontrollable such as the number of data points. Thus, controlling some of them might reduce the computational cost, represented by needed the number of outer loop iterations. The maximum number of inner loop iterations, minimum objective function improvement value, and the (m) values should be determined by this step for each instance.

Shalaby [59] suggested that the computational cost can be reduced significantly by assigning the clusters' centroids as the $1^{\text {st }}$ step of the traditional FCM algorithm for fingerprint recognition application. This assumption 
is tested for the CVRP by forcing the FCM algorithm to start with assigned initial centroids by three methods.

- Initial Centroids Based on K-means Algorithm: Kmeans clustering algorithm is one of the most wellknown partitional clustering algorithms [29]. It begins with random centroids and ends with having new centroids based on minimum distances between data points and the new centroids. The generated distance-based centroids are then used as the initial centroids for the modified FCM algorithm. The flowchart in Figure.3 represents the followed methodology of using the K-means as an initialization step for generating initial centroids.

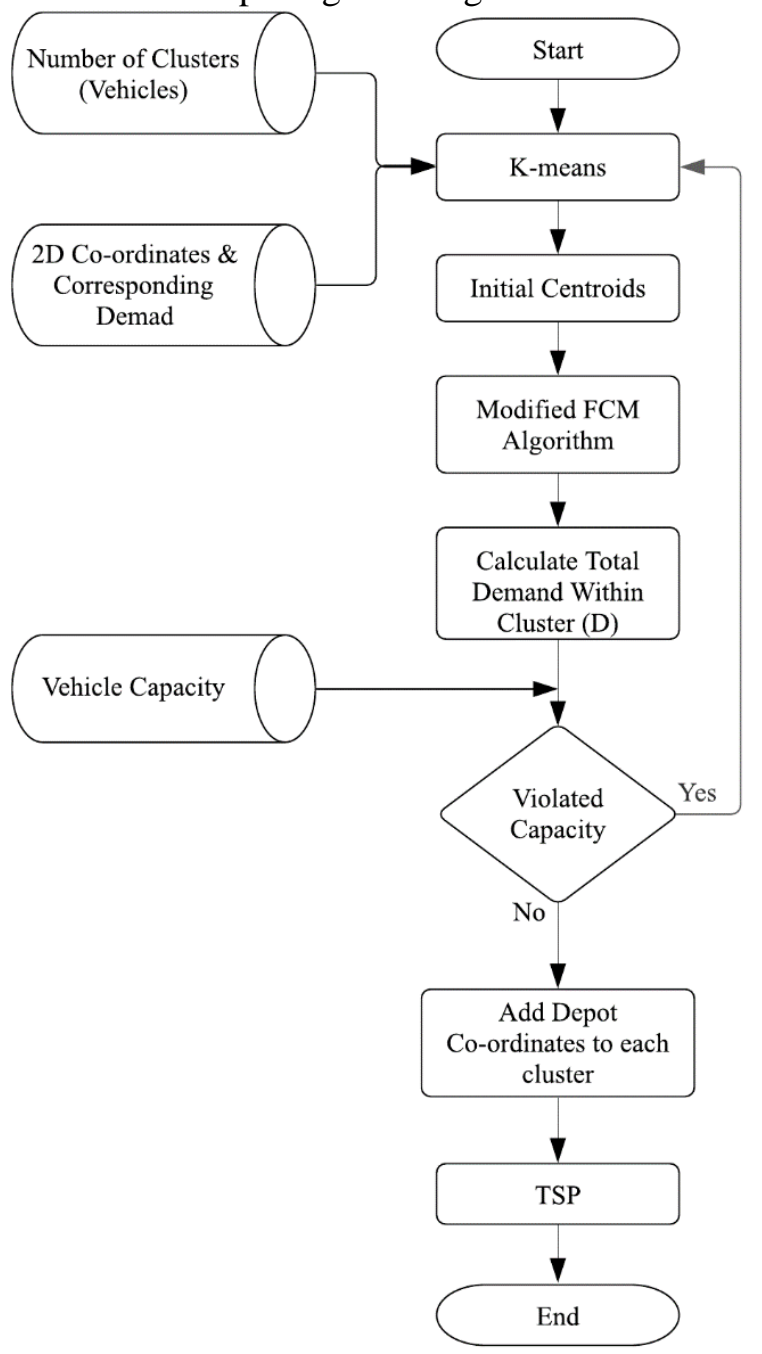

Figure 3. Flowchart of K-means for generating the initial centroids for the modified FCM.

- Initial Centroids: spatially equally distributed (SED): For the VRP problem, the cluster centers should normally be determined based on the locations of the customers to satisfy their demands and locations constraints. Hence, a good choice of initial centroids may take into consideration the spatial domain that contains the customers. In the proposed SED initialization, the boundaries of the customers'domain are first determined by knowing the maximum and minimum $x, y$ coordinates of the data points. Then the centroids are distributed equally around the center of the working domain shaping a polygon. The side length $(\mathrm{L})$ of the polygon is equal to half the distance between the domain center and the vertex of the working domain $(\mathrm{V})$. The general setup for instance P-n101-k4 is shown in Figure 4 and generalized for all the instances.

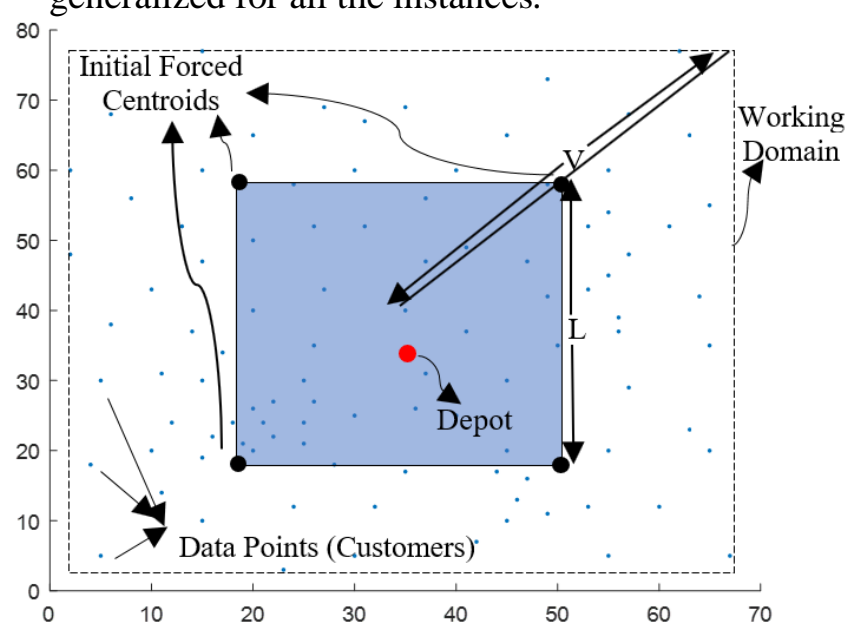

Figure 4.General setup for instance P-n101-k4 using the equal distance centroids method.

- Initial Centroids Based on Demand Weighted center of mass $(D W C M)$ : The main idea behind this technique is to expect where the centroids should be and yet save computational cost. Conveniently, the best centroids should be near the high demand customers. Thus, instead of searching the full domain, the domain is reduced based on the high demand points as follows:

$$
\begin{gathered}
W_{i}=\frac{D_{i}}{\sum_{1}^{n} D_{i}} \\
C_{X}=\sum_{1}^{n}\left(U_{i} \cdot W_{i}\right) \\
C_{Y}=\sum_{1}^{n}\left(F_{i} \cdot W_{i}\right)
\end{gathered}
$$

Where $W_{i}$ is the weighted demand of the point $i . D_{i}$ is the demand of the $i^{\text {th }}$ point. $U_{i}$ is the X-component of the point $i . F_{i}$ is the y-component of the $i^{\text {th }}$ point. $C_{X}$ is the $\mathrm{X}$ coordinate for the domain center. $C_{Y}$ is the $X$-coordinate for the domain center. Once the domain and its weighted demand center of mass are determined, the equal distance centroids method is followed again.

It is worth mentioning that in some cases the initially forced centroids lead to violated capacity. In such cases, the number of centroids is increased by one until the capacity constraint is satisfied. Adding more centroids means adding more clusters and more vehicles. This leads to increasing the overall traveled distance by the fleet and this is a limitation of using this method. The flowchart in Figure5 represents the followed methodology of the initialization step for generating initial centroids based on the proposed DWCM.

\subsection{Routing Phase}

After assigning customers to clusters the depot coordinates are added to each cluster as the trip starting 
and ending point. Each cluster is treated as a separate TSP. This is due to the nature of the VRP as an NP-hard problem while the TSP is a complete NP problem [32]. Thinkaran et al. [60] formulated the TSP with subtour elimination constraints. TSP mathematical model is available in [41].

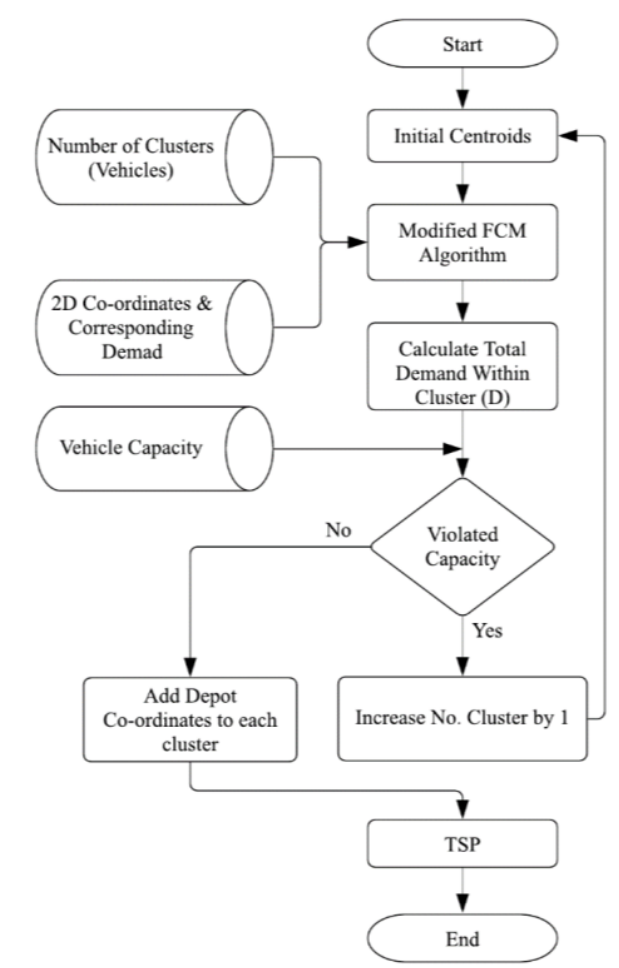

Figure 5. Flowchart generating the initial centroids for the modified FCM Based on DWCM.

\section{Computational Experimentation}

To test and verify the effectiveness of the proposed heuristics, a set of experiments are performed. The proposed heuristics are tested on 42 homogenous fleet CVRP instances from 8 different sets. Results then are compared with the optimal or best-known solutions. Sets A, B and P are developed by Augerat [3] while set $\mathrm{F}$ is developed by fisher [22]. Furthermore, sets $\mathrm{M}$ and CMT are developed by Christofides et al. [12] and set E was developed by Christofides and Elons [11]. Finally, the Golden set is developed by Golden et al. [26].

Most instances start with the name of the set that it belongs to, it is usually a letter. Then comes the number of data points in the form of the letter $n$ followed by the number without spaces. After that comes the minimum number of vehicles which is also the minimum number of clusters in the form of the letter $\mathrm{k}$ followed by the number without spaces. For example, (P-n16-k8) is an instance belonging to $\mathrm{P}$ set and has 16 data points (15 customers and the depot) requiring a minimum of 7 trucks to satisfy the demand. It is worth mentioning that the number of vehicles is calculated by dividing the total demand by the truck capacity, rounding up fractions.

Golden and CMT sets do not follow the previously stated nomenclature. In this paper, only Golden_1,
Golden_2, and Golden_3 instances are solved. Golden_1 instance has 550 data points and requires at least 9 vehicles to satisfy the demand of all customers. Furthermore, the Golden_2 instance has 320 data points including the depot requiring a minimum of 10 trucks to satisfy the whole demand. On the other hand, the CMT sets solved in this article are CMT1, CMT12, and CMT14 with $\{50,100,100\}$ data points respectively and requiring at least $\{5,10,11\}$ vehicles to satisfy the demand of all customers.

The instances are chosen to have different capacity values, demand tightness, number of customers, and number of vehicles. The number of customers varied between 31 and 399 that the number of customers is the total number of data points excluding the depot. The number of vehicles also varies between 2 and 11. The vehicle capacity varies between 35 and 30000. This variation is set to test the proposed algorithms' robustness.

Regarding FCM, there is a debate on the best fuzzy exponent $(\mathrm{m})$ to be used for a set of data. Initially, the authors used random values of $(\mathrm{m})$ and solved the problem until a good solution is obtained. Then trials to improve the solution take place by slightly increasing and decreasing the last obtained value of $(\mathrm{m})$. This technique proved to be effective for improving the initially obtained solutions.

The effect of the fuzzy exponent $(\mathrm{m})$ and how to optimize it has been studied in many previous research papers such as [8, 54]. For instance, used a genetic algorithm to optimize the parameter $(\mathrm{m})$. Okeke and Karnieli [52] used the Linear mixture model approach for selecting fuzzy exponent value. Furthermore, Choe and Jordan [10] used fuzzy decision theory for the proper selection of fuzzy exponent (m). Ewbank et al. [20] used a neural network to find optimum (m) for CVRP instances but within a discrete specific range. In this context, the authors are looking forward to developing a universal empirical equation based on the experimental data to predict the suitable (m) for CVRP. The equation should rely on average demand at each point and average distances from the depot in addition to the coefficient of variation of both the distances and demand.

The clustering process stopping criteria is when the objective function improvement is less than a specified value or achieving the maximum number of iterations. Better results can be obtained by setting a relatively high number of iterations and a low minimum objective function improvement value. Thus, the maximum number of inner loop iterations for FCM is set to be 1000 and the minimum objective function improvement value of $10^{-5}$.

Computational experiments are executed using MATLAB 2019b software on a PC with AMD Ryzen 7 3700X 8-core processor 3.6 GHz and 16.0 GB memory. Computational times for all instances range between a few seconds to a maximum of 15 minutes. At first, 
random fuzziness parameter $(\mathrm{m})$ values are used to solve the problem until a good enough solution is obtained, then the fuzziness exponent $(\mathrm{m})$ value is changed by \pm $0.1,0.3$, and 0.5 trying to obtain a better result. Each iteration involves solving each cluster as a separate TSP. The open-source code [44] used for solving the TSP depends on binary integer programming. The total distance traveled is calculated representing the CVRP solution. The code is executed 5 times for each fuzziness exponent (m) and the best result is recorded.

\section{Comparative Study and Analysis}

The unsupervised FCM algorithms and the proposed supervised FCM algorithms were applied on 42 different instances following the previously stated methodology. The best solutions were recorded for comparisons. In order to compare the obtained solution by the different techniques, three metrics have been employed, namely, deviation, total deviation, and average deviation. These metrics are calculated with respect to the optimum solution as follows:

$$
\begin{gathered}
\text { Deviation } \%=\frac{\text { Best-optimum }}{\text { optimum }} \times 100 \\
\text { Total deviation }=\frac{\sum_{1}^{25} \text { Best }-\sum_{1}^{25} \text { opt }}{\sum_{1}^{25} \text { opt }} \times 100 \\
\text { Average Deviation }=\frac{\sum \text { Deviation } \%}{25}
\end{gathered}
$$

Table 1 shows the results for each algorithm and the best result for each instance is selected as the best obtained solution. It is noticed that 33 best solutions out of the 42 are relying mainly on the modified FCM without the forced centroids in the initialization phase. This is because when we force the algorithm to start with specific centroids using any of the three techniques could limit the chances to find the best obtained solution. However, modified FCM took thousands of outer loop iterations to get best obtained solutions. On the other hand, for example, the demand weighted center of mass technique reached the same best obtained solution for 8 instances in only one iteration. This shows the significance of the forced pre-FCM initialization

\begin{tabular}{|c|c|c|c|c|c|c|c|c|}
\hline \multirow[t]{2}{*}{ Instance } & \multirow{2}{*}{ Original FCM } & \multirow{2}{*}{ Modified FCM } & \multicolumn{3}{|c|}{ Supervised Centroids Initialization } & \multirow{2}{*}{ Optimum/Best Known Value } & \multirow{2}{*}{ Best Obtained } & \multirow{2}{*}{ Deviation } \\
\hline & & & K-means initialization & Equal Distribution & Weighted Demand & & & \\
\hline A-n32-k5 & 840 & $808^{*}$ & 830 & 837 & 901 & 784 & 808 & $3.06 \%$ \\
\hline A-n33-k6 & 769 & $761^{*}$ & 765 & 769 & $761^{*}$ & 742 & 761 & $2.56 \%$ \\
\hline A-n36-k5 & 857 & $816^{*}$ & 857 & $816^{*}$ & 848 & 799 & 816 & $2.13 \%$ \\
\hline A-n33-k5 & 695 & $687 *$ & 807 & 689 & 719 & 661 & 687 & $3.93 \%$ \\
\hline A-n39-k6 & 876 & $857 *$ & 869 & 867 & 858 & 831 & 857 & $3.13 \%$ \\
\hline A-n45-k6 & 1116 & $992 *$ & 1029 & 994 & 1029 & 944 & 992 & $5.08 \%$ \\
\hline B-n31-k5 & 694 & $689^{*}$ & 700 & 693 & 709 & 672 & 689 & $2.53 \%$ \\
\hline B-n41-k6 & 888 & $860^{*}$ & 974 & 942 & 951 & 829 & 860 & $3.74 \%$ \\
\hline B-n44-k7 & 965 & $931^{*}$ & 1022 & 971 & 1056 & 909 & 931 & $2.42 \%$ \\
\hline B-n51-k7 & 1039 & $1032 *$ & 1048 & 1035 & 1039 & 1032 & 1032 & $0.00 \%$ \\
\hline B-n35-k5 & 1043 & 994* & 1052 & 996 & 1054 & 955 & 994 & $4.08 \%$ \\
\hline B-n43-k6 & 865 & $771^{*}$ & 808 & 816 & 777 & 742 & 771 & $3.91 \%$ \\
\hline B-n45-k6 & 779 & $715^{*}$ & 786 & 812 & 778 & 678 & 715 & $5.46 \%$ \\
\hline B-n38-k6 & 887 & $821^{*}$ & 860 & 880 & 864 & 805 & 821 & $1.99 \%$ \\
\hline P-n20-k2 & 241 & $232 *$ & $232 *$ & 238 & 238 & 216 & 232 & $7.41 \%$ \\
\hline P-n22-k2 & $218^{*}$ & $218^{*}$ & $218^{*}$ & $218^{*}$ & $218^{*}$ & 216 & 218 & $0.93 \%$ \\
\hline P-n21-k2 & $213^{*}$ & $213^{*}$ & $213^{*}$ & $213^{*}$ & $213^{*}$ & 211 & 213 & $0.95 \%$ \\
\hline P-n45-k5 & 535 & $518^{*}$ & 556 & 543 & 520 & 510 & 518 & $1.57 \%$ \\
\hline P-n50-k7 & 595 & $571^{*}$ & 595 & 585 & 575 & 554 & 571 & $3.07 \%$ \\
\hline P-n50-k8 & 678 & $665^{*}$ & 700 & 727 & 697 & 631 & 665 & $5.39 \%$ \\
\hline P-n55-k8 & 654 & $599^{*}$ & 642 & 654 & 614 & 576 & 599 & $3.99 \%$ \\
\hline P-n55-k7 & 603 & $584^{*}$ & 622 & 627 & 589 & 568 & 584 & $2.82 \%$ \\
\hline P-n76-k4 & 615 & $609^{*}$ & 620 & 617 & $609^{*}$ & 593 & 609 & $2.70 \%$ \\
\hline P-n76-k5 & 646 & $645^{*}$ & 771 & 653 & 655 & 627 & 645 & $2.87 \%$ \\
\hline P-n40-k5 & 663 & $468^{*}$ & 493 & 537 & 480 & 458 & 468 & $2.18 \%$ \\
\hline P-n16-k8 & 491 & $452^{*}$ & 455 & 471 & 471 & 450 & 452 & $0.44 \%$ \\
\hline P-n101-k4 & 789 & $701^{*}$ & 709 & 711 & 705 & 681 & 701 & $2.94 \%$ \\
\hline M-n101-k10 & 875 & $825^{*}$ & 829 & 860 & 829 & 820 & 825 & $0.61 \%$ \\
\hline M-n121-k7 & 1153 & $1086^{*}$ & 1130 & 1157 & 1087 & 1034 & 1086 & $5.03 \%$ \\
\hline F-n45-k4 & 925 & $736^{*}$ & $736^{*}$ & 750 & 739 & 724 & 736 & $1.66 \%$ \\
\hline F-n72-k4 & 258 & $252^{*}$ & 266 & 287 & 254 & 237 & 252 & $6.33 \%$ \\
\hline E-n22-k4 & $383^{*}$ & $383^{*}$ & 390 & 395 & $383^{*}$ & 375 & 383 & $2.13 \%$ \\
\hline E-n23-k3 & $569^{*}$ & $569^{*}$ & $569^{*}$ & $569 *$ & $569^{*}$ & 569 & 569 & $0.00 \%$ \\
\hline E-n30-k3 & 605 & $553^{*}$ & 606 & 608 & 607 & 534 & 553 & $3.56 \%$ \\
\hline E-n51-k5 & 567 & $537 *$ & 575 & 618 & 540 & 521 & 537 & $3.07 \%$ \\
\hline E-n76-k7 & 745 & $720^{*}$ & 730 & 744 & 731 & 682 & 720 & $5.57 \%$ \\
\hline CMT1 & 543 & 554 & 580 & $542 *$ & 543 & 524.61 & 542 & $3.31 \%$ \\
\hline CMT12 & $829 *$ & 837 & 838 & 876 & $829 *$ & 819.56 & 829 & $1.15 \%$ \\
\hline CMT14 & 877 & 881 & 892 & 872 & $868^{*}$ & 866.37 & 868 & $0.19 \%$ \\
\hline Golden_1 & $5774^{*}$ & 5868 & 5868 & 6342 & 6342 & 5627.54 & 5774 & $2.60 \%$ \\
\hline Golden_2 & 8876 & $8728 *$ & $8728 *$ & 9410 & 8924 & 8447.92 & 8728 & $3.32 \%$ \\
\hline Golden_3 & $11564 *$ & 11614 & 11831 & 11716 & 11720 & 11036.22 & 11564 & $4.78 \%$ \\
\hline
\end{tabular}
technique in terms of computational cost.

Table 1. Test problems and comparison of obtained results.

* Best value obtained among the FCM version.

The modified fuzzy c-means approachreaches optimum or near optimum solution for the 42 instances. The maximum deviation from optimum recorded is $7.4 \%$. The deviation percentage is calculated according to Equation (16), and the total deviation from optimum for the 42 test instances combined is $3.7 \%$, according to Equation (17), while the average deviation is $3.1 \%$, 
according to Equation (18). The results can be refined if better fuzzy exponent $(\mathrm{m})$ values are employed. It is noted that the algorithm has high efficiency and robust regardless of the size of the problem. Moreover, the solution quality is not affected by the tightness of the instances, as the maximum error recorded for the highly most tight problem (0.99 tightness) did not exceed 7.4\%. Summary calculations for all the heuristic versions are presented in Table 2.

Table 2. Comparison of modified FCM versions' results.

\begin{tabular}{|c|c|c|}
\hline Algorithm & Total Deviation & Average Deviation \\
\hline FCM & $6.5 \%$ & $8.31 \%$ \\
\hline Modified FCM & $3.7 \%$ & $3.09 \%$ \\
\hline K-means initialization & $6.6 \%$ & $7.50 \%$ \\
\hline SED initialization & $8.2 \%$ & $7.59 \%$ \\
\hline DWCM initialization & $6.7 \%$ & $5.88 \%$ \\
\hline
\end{tabular}

Some of these instances are solved in a recent study by Korayem et al. [40]. Korayem et al. used the grey wolf algorithm [44], hybridized with k-means to solve the problem. Korayem has obtained excellent results but with a limited number of customers (i.e., between 31 and 76). Besides, the capacity of vehicles did not exceed 300. The results of the proposed modified FCM approach are generally close to Korayem's results. Moreover, in a more recent study in 2019, Ewbank et al. [21] developed an improved fuzzy c-means algorithm for allocating customers to routes. This is after determining the number of clusters using a memetic algorithm. In their studies, they have solved some of the Augerat [66] instances. Again, the number of customers (i.e., between 31 and 100) and the capacity of the vehicles didn't exceed 400. Table 3 shows a comparison between the common instances solved using the proposed FCM approach and those solved in [21, 38].

It is seen from Table 3thatsome of the results obtained in [21, 38] for the common instances are slightly better than those obtained by the proposed modified FCM techniques. However, the proposed modified FCM techniques are developed straight forward based only on a clustering technique. On the other hand, the solution technique proposed in [38] is a technique that utilizes the traditional k-means clustering algorithm, hybridized with grey wolf optimizer. Furthermore, the solutions obtained from [21] are based on using fuzzy c-means along with a memetic algorithm and local search algorithm. Hence, the proposed FCMbased techniques are simpler and straightforward solution for obtaining reliable solutions for CVRP.

\section{Conclusions}

CVRP is well recognized for its importance. ClusterFirst Route-Second (CFRS) is one of the techniques widely used to solve CVRP. (FCM) clustering algorithm is known for its efficiency in solving clustering problems. This paper developed three heuristics based on the cluster-first route-second method to solve CVRP. The three proposed heuristics solved the clustering phase of CFRS using supervised FCM, then the routing phase was solved using traditional traveling salesman problem optimization methods. A modified demand weighted supervised FCM clustering algorithm was employed in the three heuristics, along with three different pre-initialization techniques, namely, K-means, spatially equal distributed, and demand weighted center of mass.

The proposed heuristics were tested on 42 instances with different characteristics. The results obtained from the three pre-FCM initialization techniques were compared with each other and with the traditional and modified unsupervised fuzzy c-means algorithms, in addition to the most recent techniques available in the literature. It was found that for the CVRP, the proposed supervised FCM techniques are capable of achieving the best obtained solution for some instances at a significant reduction of computational cost. For the remaining instances, the proposed techniques were also able to achieve reasonable solutions at the significant reduction of computational cost in comparison to other recent FCM based techniques.

Table 3. Comparison of FCM versions' results and [40, 69].

\begin{tabular}{|c|c|c|c|c|c|}
\hline \multirow{2}{*}{ Instance } & \multicolumn{2}{|c|}{ Deviation from Optimum } & \multicolumn{2}{c|}{ Difference } \\
\cline { 2 - 6 } & Best Obtained & {$[69]$} & {$[38]$} & {$[69]$} & {$[38]$} \\
\hline A-n32-k5 & $3.1 \%$ & $3.6 \%$ & - & $-0.5 \%$ & - \\
\hline A-n33-k6 & $2.6 \%$ & $1.9 \%$ & $1.6 \%$ & $0.7 \%$ & $1 \%$ \\
\hline A-n36-k5 & $2.1 \%$ & $2.1 \%$ & $1.9 \%$ & $0.0 \%$ & $0.2 \%$ \\
\hline A-n33-k5 & $3.9 \%$ & $2.9 \%$ & - & $1.0 \%$ & - \\
\hline A-n39-k6 & $3.1 \%$ & $1.7 \%$ & $0.7 \%$ & $1.4 \%$ & $2.4 \%$ \\
\hline B-n31-k5 & $2.5 \%$ & $0.6 \%$ & $0.0 \%$ & $1.9 \%$ & $2.5 \%$ \\
\hline B-n41-k6 & $3.7 \%$ & $2.7 \%$ & $1.9 \%$ & $1.0 \%$ & $1.8 \%$ \\
\hline B-n44-k7 & $2.4 \%$ & $2.1 \%$ & $3.4 \%$ & $0.3 \%$ & $-1 \%$ \\
\hline B-n35-k5 & $4.1 \%$ & $2.6 \%$ & - & $1.5 \%$ & - \\
\hline B-n43-k6 & $3.9 \%$ & $3.4 \%$ & - & $0.5 \%$ & - \\
\hline B-n38-k6 & $2.0 \%$ & $3.7 \%$ & - & $-1.7 \%$ & - \\
\hline P-n20-k2 & $5.5 \%$ & $0.9 \%$ & $0.5 \%$ & $4.6 \%$ & $5.0 \%$ \\
\hline P-n22-k2 & $0.9 \%$ & $0.5 \%$ & $0.0 \%$ & $0.4 \%$ & $0.9 \%$ \\
\hline P-n21-k2 & $0.9 \%$ & $3.8 \%$ & $0.0 \%$ & $-2.9 \%$ & $0.9 \%$ \\
\hline P-n45-k5 & $1.6 \%$ & $0.4 \%$ & $0.0 \%$ & $1.2 \%$ & $1.6 \%$ \\
\hline P-n50-k7 & $0.9 \%$ & $4.5 \%$ & - & $-3.6 \%$ & - \\
\hline P-n55-k8 & $4.0 \%$ & $3.1 \%$ & $3.1 \%$ & $0.9 \%$ & $0.9 \%$ \\
\hline P-n55-k7 & $2.8 \%$ & $12.4 \%$ & - & $-9.6 \%$ & - \\
\hline P-n76-k4 & $3.4 \%$ & $2.7 \%$ & $3.5 \%$ & $0.7 \%$ & $-0.1 \%$ \\
\hline P-n76-k5 & $2.2 \%$ & $1.4 \%$ & $1.3 \%$ & $0.8 \%$ & $0.9 \%$ \\
\hline P-n40-k5 & $2.2 \%$ & $2.2 \%$ & - & $0.0 \%$ & - \\
\hline P-n101-k4 & $2.9 \%$ & $4.1 \%$ & - & $-1.2 \%$ & - \\
\hline
\end{tabular}

\section{References}

[1] Alata M., Molhim M., and Ramini A., "Optimizing of Fuzzy C-Means Clustering Algorithm Using GA," International Journal of Computer, Electrical, Automation, Control and Information Engineering, vol. 2, no. 3, pp. 670675, 2008.

[2] Archetti C., Speranza M., and Hertz A., "A Tabu Search Algorithm for the Split Delivery Vehicle Routing Problem," Transportation Science, vol. 40, no. 1, pp. 64-73, 2006.

[3] Augerat P., Approche Polyèdrale Du Problème De Tournées De Véhicules, Doctoral dissertation, 
Institut National Polytechnique de GrenobleINPG, 1995.

[4] Barreto S., Ferreira C., Paixão J., and Santos B., "Using Clustering Analysis in A Capacitated Location-Routing Problem," European Journal of Operational Research, vol. 179, no. 3, pp. 968977, 2007.

[5] Bellman R. and Zadeh L., "Decision-Making in a Fuzzy Environment," Management Science, vol. 17, no. 4, pp. 141-164, 1970.

[6] Bezdek J., Pattern Recognition with Fuzzy Objective Function Algorithms, Plenum,1981.

[7] Bi X., Han Z., and Tang W., "Evolutionary MultiObjective Optimization for Multi-depot Vehicle Routing in Logistics," International Journal of Computational Intelligence Systems, vol. 10, no. 1, pp. 1337-1344, 2017.

[8] Bora D. and Gupta A., "Impact of Exponent Parameter Value for the Partition Matrix on the Performance of Fuzzy C Means Algorithm," Computer Vision and Pattern Recognition, vol. 3, no. 3, 2014.

[9] Bramel J. and Simchi-Levi D., The Logic of Logistics: Theory, Algorithms, and Applications for Logistics and Supply Chain Management, Springer, 2014.

[10] Choe H. and Jordan J., "On The Optimal Choice of Parameters in A Fuzzy C-Means Algorithm," inProceedings of The International Conference on Fuzzy Systems, San Diego, pp. 349-354, 1992.

[11] Christofides N. and Eilon S., "Expected Distances in Distribution Problems," Journal of the Operational Research Society, vol. 20, no. 4, pp. 437-443, 1969.

[12] Christofides N., Mingozzi A., and Toth P., "The Vehicle Routing Problem," Combinatorial Optimization, pp. 315-338, 1979.

[13] Cordeau J., Gendreau M., Hertz A., Laporte G., and Sormany J., "New Heuristics for the Vehicle Routing Problem," Logistics Systems: Design and Optimization, pp. 279-297, 2005.

[14] Cruijssen F., Bräysy O., Dullaert W., Fleuren H., and Salomon M., "Joint Route Planning Under Varying Market Conditions," The International Journal of Physical Distribution and Logistics Management, vol. 37, no. 4, pp. 287-04, 2007.

[15] Cui J., Li Q., Wang J., and Zong D., "Research on Selection Method of the Optimal Weighting Exponent and Clustering Number in Fuzzy CMeans Algorithm," in Proceedings of The International Conference on Intelligent Computation Technology and Automation, Changsha, pp. 104-107,2010.

[16] Dantzig G. and Ramser J., "The Truck Dispatching Problem," Management Science, vol. 6, no. 1, pp. 80-91, 1959.

[17] Dunn J., "A Fuzzy Relative of the ISODATA Process and Its Use in Detecting Compact Well-
Separated Clusters," Journal of Cybernetics, vol. 3, no. 3, pp. 32-57, 1973.

[18] Dunn J., "Well-Separated Clusters and Optimal Fuzzy Partitions," Journal of Cybernetics, vol. 4, no. 1, pp. 95-104, 1974.

[19] Embaby A., Shalaby M., and Elsayed K., "FCMBased Approach for Locatingvisible Video Watermarks," Symmetry, vol. 12, no. 3, pp. 33957, 2020.

[20] Ewbank H., Wanke P., and Hadi-Vencheh A., "An Unsupervised Fuzzy Clustering Approach to The Capacitated Vehicle Routing Problem," Neural Computing and Applications, vol. 27, no. 4, pp. 857-867, 2016.

[21] Ewbank H., Wanke P., Correa H., and Figueiredo O., "The Capacitated Vehicle RoutingProblem Revisited: Using Fuzzy C-Means Clustering," International Journal of Logistics Systems and Management, vol. 34, no. 4, pp. 411, 2019.

[22] Fisher M., "Optimal Solution of Vehicle Routing Problems Using Minimum K-Trees," Operations Research, vol. 42, no. 4, pp. 626-642, 1994.

[23] Gansterer M. and Hartl R., "Collaborative Vehicle Routing: A Survey," European Journal of Operational Research, vol. 268, no. 1, pp. 1-12, 2018.

[24] Gen-Yuan D., Fang M., Sheng-Li T., and Ye L., "A Modified Fuzzy C-means Algorithm in Remote Sensing Image Segmentation," in Proceedings of The International Conference on Environmental Science and Information Application Technology, Wuhan, pp. 447-450, 2009.

[25] Golden B., Assad A., Levy L., and Gheysens F., "The Fleet Size and Mix Vehicle Routing Problem," Computers and Operations Research, vol.11, no. 1, pp. 49-66, 1984.

[26] Golden B., Wasil E., Kelly J., and Chao I., "The Impact of Metaheuristics on Solving The Vehicle Routing Problem: Algorithms, Problem Sets, and Computational Results," Fleet Management and Logistics, pp. 33-56, 1998.

[27] Guajardo M. and Rönnqvist M., "A Review on Cost Allocation Methods in Collaborative Transportation," International Transactions in Operational Research, vol. 23, no. 3, pp. 371-392, 2015.

[28] Irnich S., Schneider M., and Vigo D., "Chapter 9: Four Variants of the Vehicle Routing Problem," Vehicle Routing, pp. 241-271, 2014.

[29] Jacobs-Blecha C. and Goetschalckx M., "The Vehicle Routing Problem with Backhauls: Properties and Solution Algorithms," Georgia Institute of Technology, Technical Report MHRCTR-88-13, 1993.

[30] Jayasuriya S. and Liew A., "A Modified Fuzzy CMeans Algorithm with Symmetry Information for MR Brain Image Segmentation,” in Proceedings 
of The $9^{\text {th }}$ International Conference on Information, Communications and Signal Processing, Tainan, pp. 1-5, 2013.

[31] Jiuh-Biing S., "A Hybrid Fuzzy-Optimization Approach to Customer Grouping-Based Logistics Distribution Operations,"Applied Mathematical Modelling, vol. 31, no. 6, pp. 1048-1066, 2007.

[32] Kagay S., Kikuchi S., and Donnelly R., "Use of A Fuzzy Theory Technique for Grouping of Trips in The Vehicle Routing and Scheduling Problem," European Journal of Operational Research, vol. 76, no. 1, pp. 143-154, 1994.

[33] Karakatič S. and Podgorelec V., "A Survey of Genetic Algorithms For Solving Multi Depot Vehicle Routing Problem," Applied Soft Computing, vol. 27, pp. 519-532, 2015.

[34] Karp R., "Reducibility Among Combinatorial Problems," in Proceedings of a symposium Complexity of Computer Computations, New York, pp. 219-241, 2009.

[35] Kassem S. and Mingyuan C., "A Heuristic Method For Solving Reverse Logistics Vehicle Routing Problems with Time Windows," International Journal of Industrial and Systems Engineering, vol. 12, no. 2, pp. 207-222, 2012.

[36] Kassem S., Korayem L., Khorshid M. and Tharwat A., "A Hybrid Bat Algorithm To Solve The Capacitated Vehicle Routing Problem," in Proceedings of The Novel Intelligent and Leading Emerging Sciences Conference, Giza, pp. 222225, 2019.

[37] Khaled K., Shalaby M., and El Sayed K., "Automatic Fuzzy-Based Hybrid Approachfor Segmentation, and Centerline Extraction of Main Coronary Arteries," International Journal of Advanced Computer Science and Applications, vol. 8, no. 6, pp. 258-64, 2017.

[38] Koç Ç. and Laporte G., "Vehicle Routing with Backhauls: Review and Research Perspectives," Computers and Operations Research, vol. 91, pp. 79-91, 2018.

[39] Korayem L., Khorsid M., and Kassem S., "A Hybrid K-Means Metaheuristic Algorithm to Solve A Class of Vehicle Routing Problems," Advanced Science Letters, vol. 21, no. 12, pp. 3720-3722, 2015.

[40] Korayem L., Khorsid M., and Kassem S., "Using Grey Wolf Algorithm to Solve the Capacitated Vehicle Routing Problem," IOP Conference Series: Materials Science and Engineering, vol. 83, pp.1-10, 2015.

[41] Kumar S., and Panneerselvam R., "A Survey on the Vehicle Routing Problem and Its Variants," Intelligent Information Management, vol. 4, no. 3, pp. 66-74, 2012.

[42] Lenstra J. and Kan R., "Complexity of Vehicle Routing and Scheduling Problems," Networks, vol. 11, no. 2, pp. 221-227, 1981.
[43] Marinelli M., Colovic A., and DellOrco M., "A Novel Dynamic Programming Approach for Twoechelon Capacitated Vehicle Routing Problem in City Logistics with Environmental Considerations," Transportation Research Procedia, vol. 30, pp. 147-156, 2018.

[44] Mcbratney A. and Moore A., "Application of Fuzzy Sets to Climatic Classification," Agricultural and Forest Meteorology, vol. 35, no. 1-4, pp. 165-185, 1985.

[45] Mingozzi A., Prins C., and Calvo R., "Capacitated Depot Location for the Vehicle Routing Problem," in Proceedings of the International Conference on Service Systems and Service Management, Troyes, pp. 1547-1551, 2006.

[46] Mirjalili S., Mirjalili S., and Lewis A., "Grey Wolf Optimizer," Advances in Engineering Software, vol. 69, pp. 46-61, 2014.

[47] Montoya-Torres J., Franco J., Isaza N., Jiménez H., and Herazo-Padilla N., "A Literature Review on The Vehicle Routing Problem with Multiple Depots," Computers and Industrial Engineering, vol. 79, pp. 115-129, 2015.

[48] MurugasamyK. and Mathaiyan N., "Performance Analysis of Data ClusteringAlgorithms Using Various Effectiveness Measures," The International Arab Journal of Information Technology, vol. 13, no. 6B, pp. 1084-1091, 2016.

[49] Naddef D. and Rinaldi G., "Branch-and-Cut Algorithms for the Capacitated VRP," The Vehicle Routing Problem, pp. 53-84, 2002.

[50] Narayanan S., "Travelling Salesman Problem," https://www.mathworks.com/matlabcentral/fileex change/64654-travellingsalesman-problem, Last Visited, 2020.

[51] Nasraoui O. and N'Cir C., Clustering Methods for Big Data Analytics: Techniques, Toolboxes and Applications, Springer International Publishing, 2019.

[52] Okeke F. and Karnieli A., "Linear Mixture Model Approach for Selecting Fuzzy Exponent Value in Fuzzy C-Means Algorithm," Ecological Informatics, vol. 1, no. 1, pp. 117-124, 2006.

[53] Oyola J., Arntzen H., and Woodruff L., "The Stochastic Vehicle Routing Problem, A Literature Review, Part I: Models," EURO Journal on Transportation and Logistics, vol. 7, no. 3, 193221, 2018.

[54] Papadimitriou C. and Steiglitz K., Combinatorial Optimization: Algorithms and Complexity, Mineola: Dover Publications, 2014.

[55] Pei J., Yang X., Gao X., and Xie W.,"Weighting Exponent M in Fuzzy C-Means (FCM) Clustering Algorithm," Object Detection, Classification, and Tracking Technologies International Society for Optics and Photonics, vol. 4554, pp. 246-251, 2001. 
[56] Qi C. and Sun Y., "An Improved Ant Colony Algorithm for VRPTW," in Proceedings of the International Conference on Computer Science and Software Engineering, Wuhan, pp. 455-458, 2008.

[57] Sathyanarayanan S. and Joseph K., "A Survey on Stochastic Vehicle Routing Problem," in Proceedings of theInternational Conference on Information Communication and Embedded Systems,Chennai, 2014.

[58] Shalaby M., Mohammed A., and Kassem S., "Modified Fuzzy C-Means Clustering Approach to Solve the Capacitated Vehicle Routing Problem," in Proceedings of The 21 $1^{\text {st }}$ International Arab Conference on Information Technology, $6^{\text {th }}$ of October city, pp. 1-7, 2020.

[59] Shalaby M., "Fingerprint Recognition: A Histogram Analysis Based Fuzzy C-Means Multilevel Structural Approach," Ph.D. Theses, Concordia University, 2012.

[60] Thinkaran N., Jayaprakash J., and Elanchezhian C., "Optimization of Total Cost in Inventory Routing Problem with Homogenous Type of Vehicles Using Metaheuristic Algorithm-A Review," Materials Today: Proceedings, vol. 16, pp. 1043-1047, 2019.

[61] Toth P. and Vigo D., "Models, Relaxations and Exact Approaches for The Capacitated Vehicle Routing Problem," Discrete Applied Mathematics, vol. 123, no. 1-3, pp. 487-512, 2002.

[62] Toth P. and Vigo D., The Vehicle Routing Problem, Philadelphia: Society for Industrial and Applied Mathematics, 2002.

[63] Vattani A., "K-Means Requires Exponentially Many Iterations Even in The Plane," Discrete and Computational Geometry, vol. 45, no. 4, pp. 596616, 2011.

[64] Vidal T., Crainic T., Gendreau M., Lahrichi N., and Rei W., "A Hybrid Genetic Algorithm for Multidepot and Periodic Vehicle Routing Problems," Operations Research, vol. 60, no. 3, pp. 611-624, 2012.

[65] Wolsey L., Integer Programming, Wiley, 1998.

[66] Xu X., Lin Z., and Zhu J., "DVRP with Limited

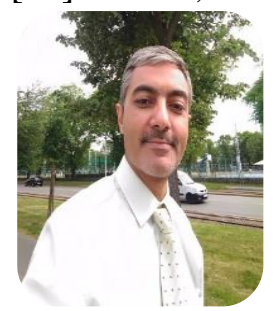
Supply and Variable Neighborhood Region in Refined Oil Distribution," Annals of Operations Research, 2020.

[67] Yalcin G. and Erginel N., "Fuzzy Multi-Objective Programming Algorithm for Vehicle Routing Problems with Backhauls," Expert Systems with Applications, vol. 42, no. 13, pp. 5632-5644, 2015.

[68] Yang M., "A Survey of Fuzzy Clustering," Mathematical and Computer Modelling, vol. 18, no. 11, pp. 1-16, 1993.
[69] Yang W., Mathur K., and Ballou R., "Stochastic Vehicle Routing Problem with Restocking," Transportation Science, vol. 34, no. 1, pp. 99-112, 2000.

[70] Yong W., Ma X., Xu M., Wang Y., and Liu Y., "Vehicle Routing Problem Based on A Fuzzy Customer Clustering Approach for Logistics Network Optimization," Journal of Intelligent and Fuzzy Systems, vol. 29, no. 4, pp. 1427-1442, 2015.

Mohamed Shalaby is an IEEE member, he received the $\mathrm{PhD}$ degree in electrical and computer engineering from Concordia University, Canada in 2012. Starting from 2013 till 2017, he was a full-time assistant professor in the fields of robotics, image and video processing at the information technology department, faculty of computers and artificial intelligence, Cairo University, Giza, Egypt. Then he joined the mechatronics program at faculty of engineering and applied sciences, Nile University, Giza, Egypt. His research interests include robotics, artificial intelligence, deep learning, biometrics, image processing, pattern recognition, and 
computer vision. Dr. Shalaby has been an associate editor of the Egyptian Informatics Journal, Elsevier, since 2015. Recently, Dr. Shalaby has been a founding member of the multi-disciplinary Smart Engineering Systems Research Center (SESC) at Nile University, Giza, Egypt.

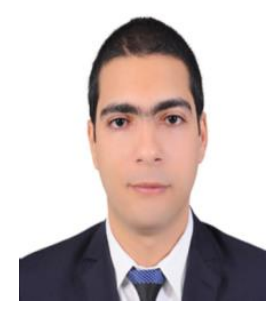

Ayman Mohammed is a young researcher who obtained his BSc in Industrial Engineering from the school of engineering and applied sciences, Nile University in 2020. During his undergraduate studies at Nile University, he has understood and practiced the basics of scientific research in the field of industrial engineering and management. $\mathrm{He}$ is currently a MSc Mechatronics engineering student and works as a research assistant at Smart Engineering Systems Research Center (SESC).
School of Engineering and Applied Sciences, Industrial and Service Engineering Program, Nile University, Egypt. Dr. Sally Kassem co-authored several research papers that have been published in reputable journals and conferences, in diverse fields, for example, logistics and supply chain, IOT monitoring optimization, design for sustainability, modeling and simulation. She served as a reviewer for a number of international conferences and journals of well-recognized publishers and organizations, like Elsevier, IEEE, and Inderscience.

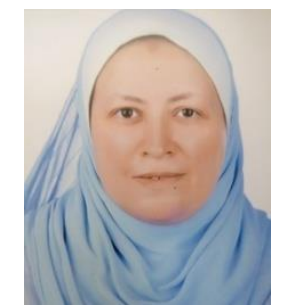

Sally Kassem obtained her B.Sc. degree from mechanical design and production department, Faculty of Engineering, Cairo University in 1998. She earned her MSc. Degree in industrial engineering at the same affiliation, and the Ph.D. degree in industrial engineering in 2011 at the Department of Mechanical and Industrial Engineering, Concordia University, Montreal, Canada. Dr. Kassem has been an assistant professor at the Department of Operations Research and Decision Support, Faculty of Computers and Information, Cairo University since 2013. Currently, Dr. Kassem is an assistant professor at the 